\title{
Erratum to: A Big Data Framework for Early \\ Identification of Dropout Students in MOOC
}

\author{
Jeff K.T. Tang ${ }^{1}$, Haoran Xie ${ }^{2}$, and Tak-Lam Wong ${ }^{3(\bowtie)}$ \\ ${ }^{1}$ School of Computing and Information Sciences, \\ Caritas Institute of Higher Education, Hong Kong, China \\ jtang@cihe.edu.hk \\ ${ }^{2}$ Centre for Excellence, Caritas Institute of Higher Education, \\ Hong Kong, China \\ hrxie2@gmail.com \\ 3 Department of Mathematics and Information Technology, \\ The Hong Kong Institute of Education, Hong Kong, China \\ tlwong@ied.edu.hk
}

\section{Erratum to: \\ Chapter 12: J. Lam et al. (Eds.) \\ Technology in Education \\ DOI: 10.1007/978-3-662-48978-9_12}

In the original version, the name of the second author was spelled incorrectly by mistake. It should be Haoran Xie. 